\title{
Hamartoma pulmonar nodular periférico en una paciente con artritis reumatoide
}

\section{Peripheral nodular pulmonary hamartoma in a patient with rheumatoid arthritis}

\author{
Ludmila Cosío-Lima ${ }^{1 *}$ y Miguel Cosío-Pascal ${ }^{2}$ \\ ${ }^{1}$ Departamento de Las Ciencias del Movimiento y Salud, Universidad de West Florida, Pensacola, Florida, Estados Unidos de América; ${ }^{2}$ Unidad \\ de Neumología, Hospital Ángeles Mocel, Ciudad de México, México
}

\begin{abstract}
Resumen
Reportamos un caso de una joven de 23 años que ante la reactividad de su artritis nos fue solicitada nuestra opinión acerca del caso. Después de examinarla y encontrar un nódulo en el lóbulo pulmonar derecho, lo más importante era determinar si el nódulo era tuberculoso y si aún estaba activo. Por las imagenologías nos pareció que no era tuberculoso. Se recomendó a la paciente que se operara.
\end{abstract}

Palabras clave: Artritis reumatoide. Nódulo pulmonar. Hamartoma.

\section{Abstract}

We report a case of a 23-year-old woman who was referred to us due to her side effects of her arthritis medications. We detected a nodule located in the right pulmonary lobule. The most important thing was to determine if this nodule was tuberculous and to know if it was still active. From the imaging the nodule seemed not to be tuberculous. We recommend that she proceeded with surgery.

Key words: Rheumatoid arthritis. Pulmonary nodule. Hamartoma.

\section{Introducción}

Los hamartomas se conocen desde la antigüedad; su nombre proviene del griego: hamartia (defecto) y oma (tumor). Se consideran formaciones benignas que simulan una neoplasia del tejido en donde se originan ${ }^{1}$. Sin embargo, se ha demostrado que algunos pueden mostrar aberración clonal originada por mutaciones aberrantes, resultando en tumores malignos ${ }^{2,3}$.

Los hamartomas generalmente son nódulos pequeños y crecen en cualquier tejido; son asintomáticos la mayoría de ellos, aunque según su localización y tamaño pueden producir sintomatología en ocasiones muy grave. Se componen de elementos que se encuentran en el tejido normal donde aparecen, pero crecen en forma desorganizada. En la mayoría de las ocasiones, cuando son asintomáticos son descubiertos incidentalmente por imagenología practicada para estudiar otra patología. Son considerados por algunos investigadores como hamartomas y por otros como neoplasias benignas ${ }^{4}$.
Correspondencia:

*Ludmila Cosío-Lima

E-mail: Icosiolima@uwf.edu
Disponible en internet: 13-11-2020 Rev Hosp Jua Mex. 2020;87(3):109-111

www.revistahospitaljuarez.com 1405-9622/○ 2020 Sociedad Médico-Quirúrgica del Hospital Juárez de México, A.C. Publicado por Permanyer. Este es un artículo open access bajo la licencia CC BY-NC-ND (http://creativecommons.org/licenses/by-nc-nd/4.0/). 
Los hamartomas más frecuentes se localizan en los pulmones. Aproximadamente el $3-8 \%$ de los nódulos pulmonares son hamartomas originados a partir de tejido conectivo, cartílago y tejido graso ${ }^{4}$. El $10 \%$ de ellos se relacionan con la tráquea o bronquios. Los endobronquiales hacen pensar en principio en un carcinoma broncogénico: tos, expectoración, sangrado, atelectasia, etc., siendo generalmente candidatos a cirugía ${ }^{4}$. Tajima, et al. ${ }^{5}$ reportan un caso tratado con éxito vía irradiación con láser Nd-YAG (neodymium-doped yttrium aluminium garnet).

En algunos casos se identifican calcificaciones. Los hamartomas periféricos son asintomáticos. La imagenología en ocasiones no los clasifica fácilmente, a menos que se detecte cartílago o la clásica imagen en «palomita de maíz». Green ${ }^{6}$ reporta hallazgos incidentales en tomografías de tórax en los nódulos periféricos. Hughes ${ }^{7}$ plantea la posibilidad de realizar biopsia del nódulo con aguja fina, valorando los resultados. Gjevic $^{8}$ analiza y relata la patología en 215 pacientes con hamartoma pulmonar en la Clínica Mayo.

\section{Presentación del caso}

Paciente femenina de 23 años de edad sin antecedentes familiares de interés, que ha estado en tratamiento en relación con la artritis reumatoide a base de metrotexato, hidroxicloroquina, ácido fólico, e inhibidor del factor de necrosis tumoral, habiendo estado razonablemente controlada. Las radiografías de tórax (Fig. 1) mostraron un nódulo de $3 \mathrm{~cm}$ situado en la periferia del lóbulo pulmonar derecho. Su médico tratante, ante la posibilidad de que el nódulo fuera tuberculoso y activo, le prescribió rifampicina como tratamiento profiláctico durante siete meses. Al empeorar la paciente y juzgando que necesitaba un cambio más enérgico de inmunosupresores es referida al área de Neumología del Hospital General de México para una opinión acerca de la actividad del nódulo.

En Neumología se efectuó una historia clínica y un examen físico rigurosos, incluyendo antecedentes de tuberculosis, habito tabáquico o exposición a riesgos laborales 0 asbestos y coexistencia de infecciones sistémicas específicas. Tras haber observado las radiografías, el nódulo y haber confirmado que no era tuberculoso, pero sin tener un diagnóstico fijo, se decidió efectuar la resección del nódulo.

\section{Procedimiento quirúrgico}

El nódulo estaba localizado en el lóbulo inferior del pulmón izquierdo y muy cerca de la pared del tórax. La

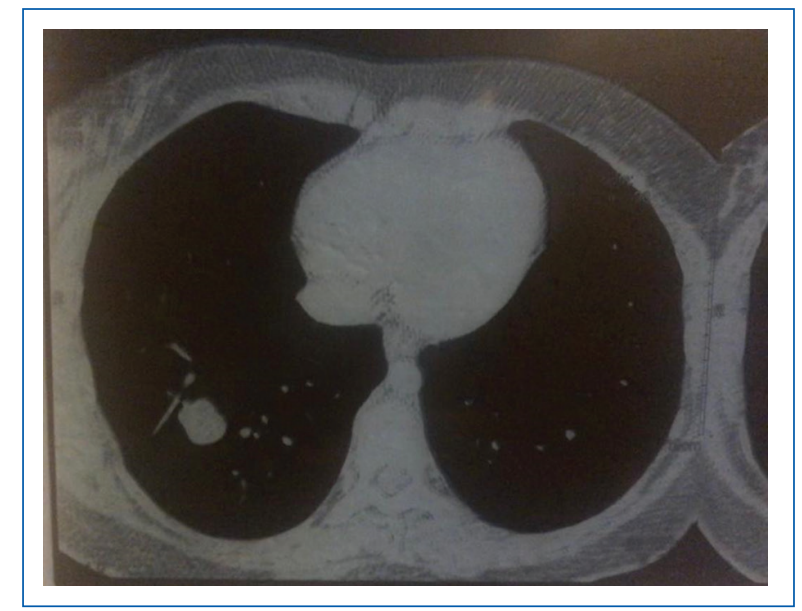

Figura 1. La radiografía de tórax con un nódulo de $3 \mathrm{~cm}$ situado en la periferia del lóbulo pulmonar derecho.

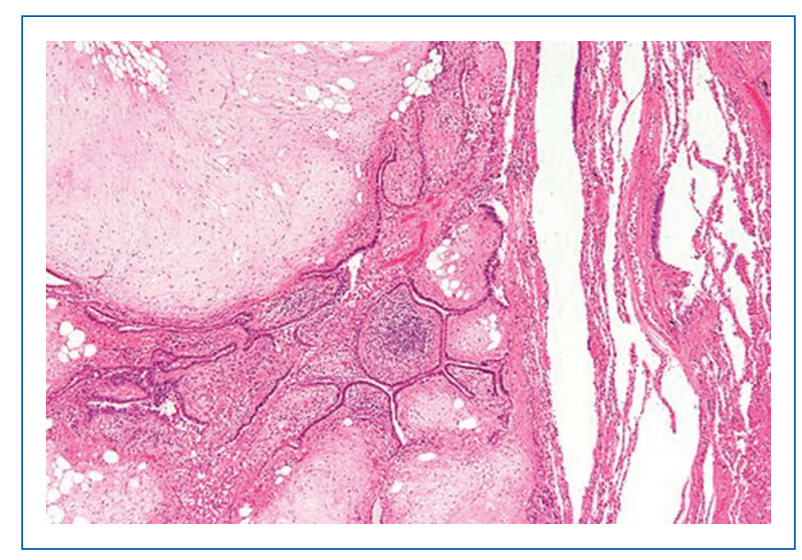

Figura 2. Histología. En la laminilla se identifican tejido conectivo, tejido graso y la presencia principalmente de cartílago.

paciente fue colocada en decúbito lateral izquierdo y bajo anestesia general se realizó el colapso selectivo del pulmón derecho. Tres trócares fueron colocados en la línea axilar posterior, dos en el quinto y séptimo espacios intercostales y uno en el séptimo espacio intercostal. El nódulo fue visualizado con el toracoscopio y con una engrapadora GIA $35 \mathrm{~mm}$ se hizo una resección en cuña con dos disparos. El nódulo se extrajo con una bolsa y fue enviado a analizar. Se colocó el tubo de pleurostomía, se cerraron las incisiones y la paciente fue extubada sin complicaciones. La histología final del tumor (Fig. 2) fue un hamartoma pulmonar con predominio de tejido adiposo y diferenciación leiomiomatoso. El nódulo ya resecado presenta el típico aspecto de «palomita de maíz» (Fig. 3). El resultado del informe resultó ser un hamartoma pulmonar periférico. 


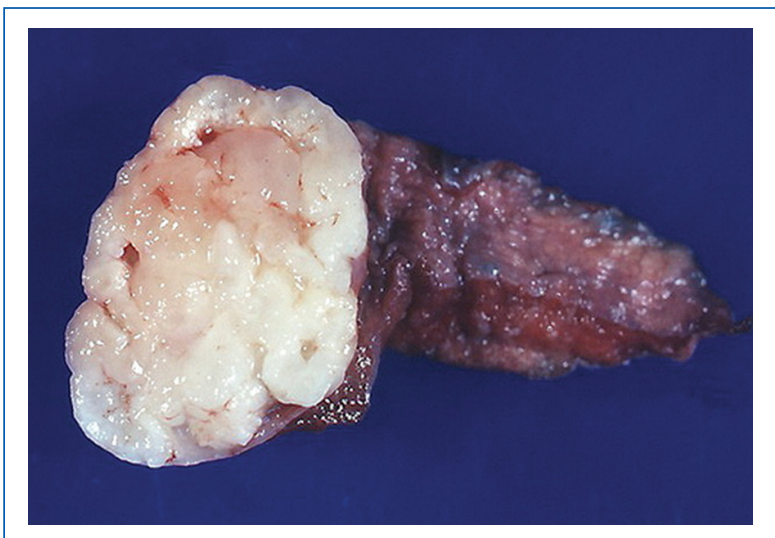

Figura 3. El nódulo resecado muestra el típico aspecto de "palomitas de maíz».

\section{Evolución}

La recuperación postoperativa fue un éxito, sin disnea y mínimo dolor, por lo que el tubo pleural fue retirado después de $24 \mathrm{~h}$ y la paciente fue dada de alta a su casa después de dos días de estancia en el hospital. Seis meses después de ser dada de alta la paciente sigue bien y sin problemas.

\section{Conclusión}

Los hamartomas benignos pueden causar serios problemas, como serían principalmente la localización y tamaño comprimiendo, obstruyendo bronquios o desplazando estructuras vecinas. Joshi, et al. describen un caso de hamartoma pulmonar torácico gigante que provocó falla ventricular derecha ${ }^{9}$. Okabayashi ${ }^{10}$ describe un hamartoma gigante en tórax que producía carbohidrato antígeno 9-9.

Como se mencionó, esta patología puede aparecer en cualquier sitio, siendo asintomático o complicando la función del órgano donde se localice. Cuando aparece en el rostro, la deformidad de la cara es impresionante. En nuestro caso, después de obtener el diagnóstico correcto en la paciente asintomática, la cirugía fue muy sencilla, mediante resección localizada.

Por último, nos referiremos brevemente a los síndromes de polinosis amargosas:

- Síndrome de Codeen o de hamartosis múltiple. Es de origen genético trasmitido por patrón autosómico.

Se caracteriza por múltiples localizaciones.

- Síndrome de Peutz-Jeghers. Numerosos hamartomas diseminados y pigmentados en piel.
- Síndrome de Cowden o de hamartosis múltiples, también de origen genitivo y se caracteriza por la aparición de múltiples hamartomas indiferentes localizaciones, sobre todo en tracto gastrointestinal. Los tres síndromes revisten importante gravedad.

\section{Financiamiento}

La presente investigación no ha recibido ayudas específicas provenientes de agencias del sector público, sector comercial o entidades sin ánimo de lucro.

\section{Conflicto de intereses}

Los autores declaran no tener conflicto de intereses alguno.

\section{Responsabilidades éticas}

Protección de personas y animales. Los autores declaran que los procedimientos seguidos se conformaron a las normas éticas del comité de experimentación humana responsable y de acuerdo con la Asociación Médica Mundial y la Declaración de Helsinki.

Confidencialidad de los datos. Los autores declaran que han seguido los protocolos de su centro de trabajo sobre la publicación de datos de pacientes.

Derecho a la privacidad y consentimiento informado. Los autores han obtenido el consentimiento informado de los pacientes y/o sujetos referidos en el artículo. Este documento obra en poder del autor de correspondencia.

\section{Bibliografía}

1. Mandewell JE, Feigin DS. Bening tumors of the lung. Semin Roentgenol. 1977;12:175-86.

2. Poulsen JT, Jacobsen $M$, Francis $D$. Probable malignant transformation of a pulmonary hamartoma. Thorax. 1979;34(4):557-8.

3. Ribet M, Jaullard TS, Nuttens MC. Pulmonary hamartoma and malignancy. J Thorac Cardiovasc Surg. 1994;107(2):611-4.

4. Baetson EM. Relationship between intrapulmonary and endobronchial cartilage containing tumors (so called hamartomas). Thorax. 1965;20: 447-61.

5. Tajima H, Hayashi $\mathrm{Y}$, Maehara T, Morohoshi T, Imada T, Amano T, et al. Endobronchial hamartoma treated by an Nd-YAG laser: report of a case. Surg Today. 1998;28(10):1078-80.

6. Green D. Incidental findings computed tomography of the thorax. Semin Ultrasound CT MR. 2005;26(1):14-9.

7. Hughes JH, Young NA, Wilbur DC, et al. Fine-needle aspiration of pulmonary hamartoma: A common source of false-positive diagnoses in the College of American Pathologists Interlaboratory Comparison Program in Nongynecologic Cytology. Arch Pathol Lab Med. 2005;129(1):19-22.

8. Gjevre JA, Myers JL, Prakash UB. Pulmonary hamartomas. Mayo Clin Proc. 1996;71(1):14-20.

9. Joshi HM, Page RD. Giant pulmonary hamartoma causing acute right heart failure. Ann Thorac Surg. 2014;97(1):e21-2.

10. Okabayashi K, Hiratsuka M, Noda Y, Hanagiri T, Mitsudomi T, Shirakusa T, et al. Giant hamartoma of the lung with a high production of carbohydrate antigen 19-9. Ann Thorac Surg. 1993;55(2):511-3. 